\title{
Dissecting mechanisms regulating the ocean Fe cycle by an unsupervised classification
}

\author{
Anh Le-Duy Pham ${ }^{1,2}$ and Takamitsu Ito ${ }^{1}$
}

1. School of Earth and Atmospheric Sciences, Georgia Institute of Technology, Atlanta, USA

2. Laboratory of Oceanography and Climate: Experiments and Numerical Approaches (LOCEAN), Paris, France

Recent collaborative efforts from the GEOTRACES program have rapidly expanded the dataset of the micronutrient dissolved iron (dFe) distribution in the global ocean, which provides a unique opportunity to improve our understanding of the ocean iron cycling. Previous modeling and observational studies focused on constraining the magnitude of external dFe inputs into the ocean. However, biochemical processes within the water column also affect observed dFe concentrations, and their interactions with diverse dFe sources are not yet fully understood. Here we evaluated the relative roles of external dFe sources versus interior ocean processes by performing a series of computational experiments and by analyzing the simulated dFe mass balance through an unsupervised machine learning technique. The results demonstrate that the upper ocean $\mathrm{dFe}$ gradient is regulated by biochemical processes in the interior ocean, where the delicate balance between scavenging and dissolution of Fe is modulated by the availability of organic ligands. While external dFe inputs set the overall magnitude for the ocean dFe concentration, biochemical processes regulate the ocean $\mathrm{dFe}$ pattern. 